\title{
Sleep and drug resistant epilepsy in children
}

Keywords: seizures, hypersomnia, neuropsychiatric, carbamazepine, levetiracetam

Abbrevations: OSA, Obstructive Sleep Apnea; SUDEP, VNS, vagus nerve stimulation; sudden death in epilepsy; SRBD, Sleep Related Breathing Disorders; ESES, electrical status epilepticus of sleep

\section{Introduction}

According to ILAE drug-resistant epilepsy is defined as a failure of adequate trials of 2 tolerated and appropriately chosen and used AED schedules. ${ }^{1}$ In addition to suffering intractable epileptic seizures, children with drug resistant epilepsy suffer from co-morbid illnesses like cognitive problems, neuropsychiatric issues, disorders of bone metabolism and sleep abnormalities. Sleep problems in intractable epilepsy are often neglected. Studies have shown high prevalence of sleep disturbances like Obstructive Sleep Apnea Syndrome (OSA), Excessive Daytime Sleepiness, Hypersomnia, Narcolepsy, Periodic Limb Movement Disorder and Restless Leg Syndrome in Childhood epilepsy. ${ }^{2}$ This review expounds the intricate and reciprocal association between sleep and drug resistant epilepsy in children.

Factors contributing to sleep disturbance in drug resistant epilepsy

There are multiple factors which contribute to sleep problems in drug resistant epilepsy. The most important of all seems to be the abnormal macro and micro-architecture of sleep due to epilepsy itself. The recurrent seizures have a dominant effect on sleep architecture, particularly during the post-ictal phase. Type of epileptic syndrome, seizure type, extent of neurological involvement and administration of antiepileptic drugs may be associated with alterations in quality of sleep. ${ }^{3}$ Alterations in the neuronal networks responsible for sleep wake cycle regulation may play a significant role. Antiepileptic affect sleep in multiple ways. Benzodiazepines can cause excessive day time sleepiness. Valproate may contribute to weight gain which in turn can progress to OSA in extreme cases. Carbamazepine and levetiracetam may result in excessive limb movements. Lamotrigine and pregabalin may cause insomnia. ${ }^{4}$ Patients with intractable epilepsy may have insufficient sleep time and poor sleep hygiene, particularly when the seizures are frequent. Sleep habits in children with intractable epilepsy are frequently affected due to parental difficulties like fear of missing, not recognizing a nocturnal seizure or sudden death in epilepsy (SUDEP).

\section{Complex association between sleep and epilepsy}

Sleep deprivation has seizure provoking effects which may be due to disruption of hypothalamically mediated circadian regulation. Frequent and recurrent seizures may in turn lead to a vicious cycle of sleep fragmentation. Occurrence of seizures during wakefulness in the day time can affect the nocturnal sleep. Different stages of sleep particularly stage II may trigger seizures as well as interictal epileptiform discharges and can also modify the morphology of epileptiform discharges. The occurrence of seizures during sleep is

\author{
Volume 2 Issue 4 - 2018
}

\author{
Ranjith Kumar Manokaran, Sheffali Gulati \\ Department of Pediatrics, All India Institute of Medical Sciences, \\ India
}

Correspondence: Sheffali Gulati, Chief, Child Neurology Division, Department of Pediatrics, All India Institute of Medical Sciences, New Delhi, India Email sheffalig@gmail.com

Received: February 25, 2018| Published: August 09, 2018

caused by an intimate relationship between the physiological state of sleep and the pathological process underlying epileptogenesis. Sleep Related Breathing Disorders (SRBD) can be a potential trigger for paroxysmal activity and interictal epileptiform discharges. Sleep disorders such as obstructive sleep apnea or restless leg syndrome may influence seizure control and effective treatment of sleep disorders can improve seizure control.

Sleep deprivation is a precipitant factor in juvenile myoclonic epilepsy. Natural sleep may facilitate the appearance of generalized seizures or epileptiform discharges, while sleep deprivation may accentuate the yield of EEG abnormality in partial epilepsy. Sleep organization in West syndrome and Lennox-Gastaut Syndrome is profoundly disrupted. Electrical status epilepticus of sleep (ESES) and Landau-Kleffner syndrome are forms of epilepsy in which abundant epileptiform discharges are present during slow wave sleep (Figure 1A) (Figure 1B) (Figure 2). In benign childhood epilepsy with centrotemporal spikes, there is an accentuation of centrotemporal spikes during sleep (Figure 3).

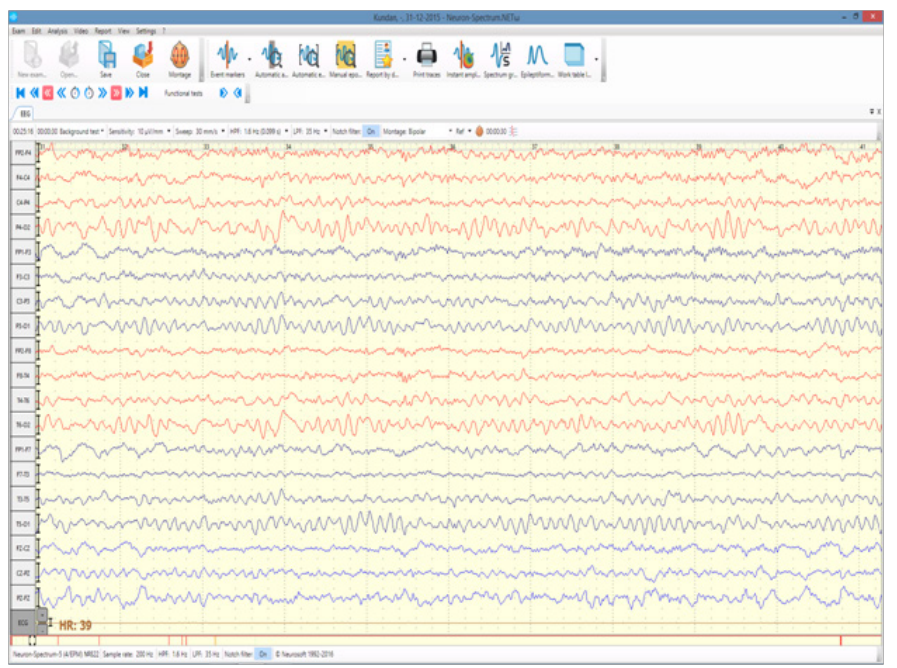

Figure I(A) 8year old developmentally normal till 4 years of age presented with behavioral problems, hyperactivity, aggressiveness, motor deficits with gait instability and seizures (GTCS/ Absences).Normal awake EEG record of the patient. 


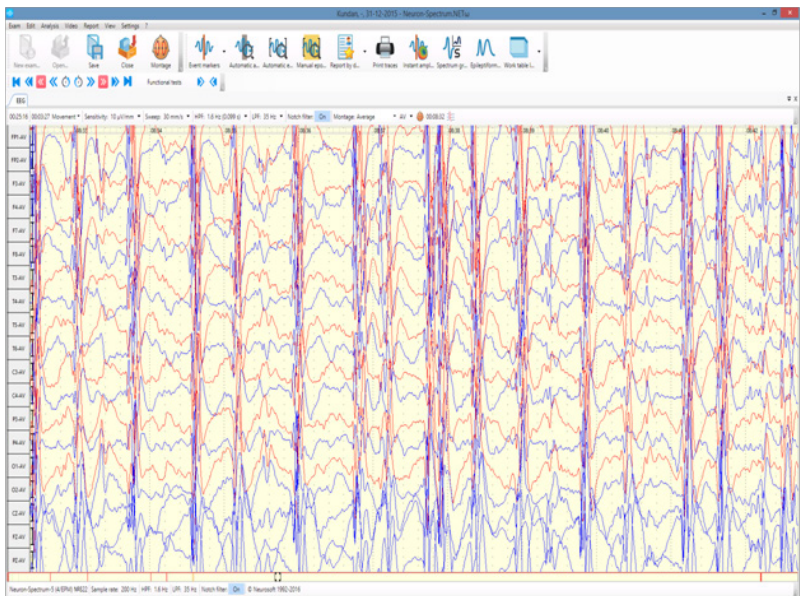

Figure I(B) Sleep EEG record of the same patient shows generalized I-2 Hz, spike wave discharges with front central predominance suggestive of electrical status epilepticus of sleep (ESES).

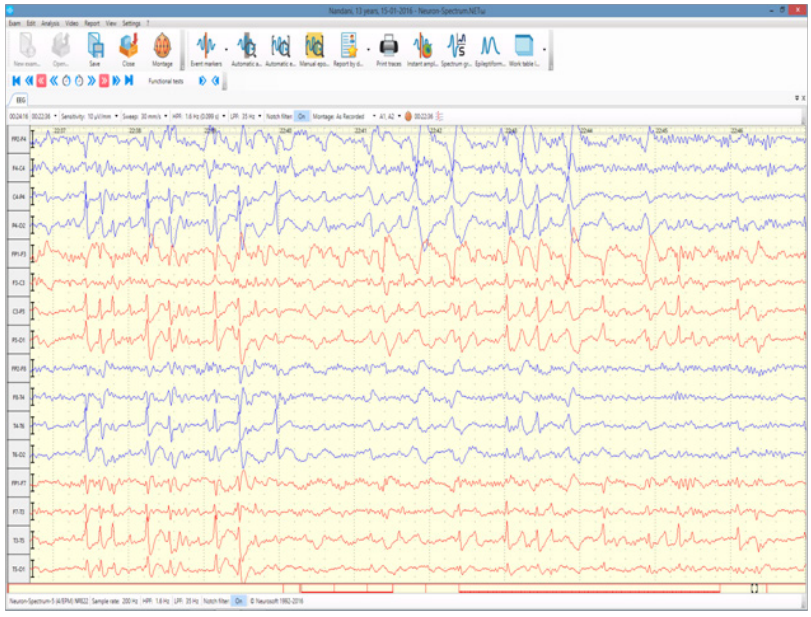

Figure 2 7year old developmentally presented with autistic regression and word deafness. There was no history of any seizures. Sleep EEG record of the patient shows abundant bi temporal spikes and spike wave discharges in sleep suggestive of Landau Kleffner syndrome.

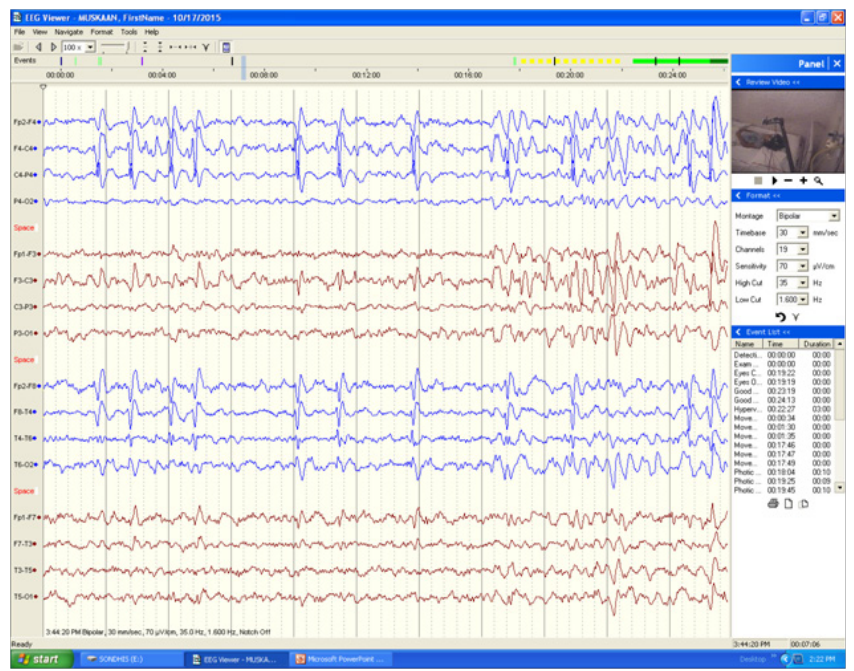

Figure $39 y e a r$ old school going child presented with nocturnal focal seizures involving face associated with guttural sounds and drooling. Sleep EEG record shows runs of bilateral centrotemporal spikes with a horizontal dipole suggestive of benign rolandic epilepsy.

\section{Quality of sleep in children with drug resistant epilepsy}

Patients with epilepsy generally have sleep macrostructure abnormalities, such as increased sleep onset latency, increased number and duration of awakenings, reduced sleep efficiency, reduced or abnormal $\mathrm{K}$ complexes and sleep spindles, reduced or fragmented rapid eye movement (REM sleep), and increased stage shifts. ${ }^{5}$ Nunes et al., ${ }^{5}$ evaluated sleep organization in pediatric patients with partial refractory epilepsy by polysomnography. They showed there was a reduction of total time in bed and total sleep time. The percentage of stage 2 was significantly reduced in patients with epilepsy that presented seizures during night recordings and percentage of stages 3-4 was increased. ${ }^{6}$ About $45 \%$ of patients with medically refractory epilepsy experienced excess daytime sleepiness .?

Kaleyias et al., ${ }^{8}$ reported a high percentage (80\%) of sleep disruption because of either obstructive apnea syndrome, disturbance of sleep architecture, or sleep fragmentation in children with epilepsy. ${ }^{8}$ Sleep studies in children with epilepsy have also shown reduced sleep efficiency, heightened anxiety concerning their night time sleep, higher incidence of behavioral problems and worse neuropsychologic functioning. In a study by Nunes et al., ${ }^{6}$ it was found that children with epilepsy had significantly worse sleep habits than controls considering night timebehaviors, presence of night-waking, parental guidelines for bedtime and rise-time expectations. ${ }^{6}$ Sleep abnormalities are more frequent in patients who have drug resistant epilepsy. Post ictal sleepiness and confusion are very common in children with Dravet syndrome and Lennox Gastaut Syndrome when there is intractable epilepsy. Now with the recent FDA approval of Cannabidiol derived Epidiolex, there may be some hope on the horizon for these conditions with intractable epilepsy. Children with poor seizure control had significantly lower sleep efficiency, a higher arousal index, and a higher rapid eye movement percentage compared with children who were seizure-free or children with good seizure control.

Intermittent vagus nerve stimulation (VNS) can reduce the frequency of seizures in patients with drug resistant epilepsy, but can affect breathing in sleep. Untreated obstructive sleep apnea (OSA) can worsen seizure frequency. Unfortunately, OSA and VNS-induced sleep disordered breathing (SDB) may occur in the same patient, leading to a therapeutic dilemma. Epileptologists need to balance seizure control and SDB in patients with VNS. ${ }^{9}$

\section{Management of sleep problems}

Disruption of sleep architecture and sleep deprivation can lead to lowering of seizure threshold. Sleep abnormalities can lead to excessive day time somnolence and hence subsequently affect neuropsychologic development, memory, behavior, cognition and mood. Failure to recognize and treat sleep disturbances can lead to worsening of intellect, cognitive functioning, and quality of life, and can also increase seizure frequency. Age appropriate sleep questionnaires can be used. Excessive day time somnolence can be assessed by modified pediatric Epworth sleepiness scale. Polysomnography may be performed in indicated cases.

Regularizing sleep habits by advising proper sleep hygiene can be beneficial. Optimization of anti-epileptic drugs (avoiding agents causing sleep disturbances) is another important strategy. Behavioral therapy can be extended to the patients. Treatment of OSA with tonsillo-adenoidectomy can be beneficial in select patients. Recent evidence suggests the use of melatonin in intractable epilepsy was associated with improvement of both many sleep-related phenomena and the severity of seizures.$^{10}$ Hall brook et al. ${ }^{11}$ showed recently that ketogenic diet improves sleep quality in children with drug resistant 
epilepsy and provides additional benefits including behavioral improvements. ${ }^{11}$ Adequate control of seizures during sleep (especially generalized tonic-clonic seizures) decreases risk of sudden unexpected death in epilepsy (SUDEP).

\section{Conclusion}

Epilepsy and sleep are reciprocally related to each other; seizure control will improve the sleep quality while improving sleep will help in achieving seizure control. Children with drug resistant epilepsy, even without intellectual disability or epileptic encephalopathy, have an increased prevalence of sleep complaints, sleep disorders and daytime sleepiness. In this review, we have elucidated few case vignettes to show the intricate relationship between the sleep and epilepsy. It is the need of the hour for a larger prospective study evaluating the sleep problems in epilepsy and objectively examines them by polysomnographic parameters. Proper identification and management of sleep problems can help improve seizure control and overall quality of life.

\section{Acknowledgements}

None.

\section{Conflict of interest}

The author declares that there are no conflicts of interest.

\section{References}

1. Kwan P, Arzimanoglou A, Berg AT, et al. Definition of drug resitant epilepsy: Consensus proposal by the ad hoc task force of the ILAE commission on therapeutic strategies. Epilepsia. 2010;51(6):1069-1077.
2. Kotagal S, Yardi N. The relationship between sleep and epilepsy. Semin Pediatr Neurol. 2008;15(2):42-49.

3. Kothare SV, Kaleyias J. Sleep and epilepsy in children and adolescents. Sleep Med. 2010;11(7):674-685.

4. Foldvary N, Perry M, Lee J, et al. The effects of lamotrigine on sleep in patients with epilepsy. Epilepsia: 2001;42(12):1569-1573.

5. Nunes ML. Sleep and epilepsy in children: Clinical aspects and polysomnography. Epilepsy Res. 2010;89(1):121-125.

6. Nunes ML, Ferri A, Arzimanoglou A, et al. Sleep organization in children with partial refractory epilepsy. J Child Neurol. 2003;18(11):763-766.

7. Foldvary Schaefer N, Grigg Damberger M. Sleep and epilepsy. Semin Neurol. 2009;29(4):419-428.

8. Kaleyias J, Cruz M, Goraya JS, et al. Spectrum of polysomnographic abnormalities in children with epilepsy. Pediatr neurol. 2008:39(3):170-176.

9. Upadhyay $\mathrm{H}$, Bhat $\mathrm{S}$, Gupta $\mathrm{D}$, et al. The therapeutic dilemma of vagus nerve stimulator-induced sleep disordered breathing. Ann Thorac Med. 2016;11(2):151-154.

10. Elkhayat HA, Hassanein SM, Tomoum HY. Melatonin and SleepRelated Problems in Children with Intractable Epilepsy. Pediatr Neurol. 2010;42(4):249-254.

11. Hallböök T, Lundgren J, Rosén I. Ketogenic Diet Improves Sleep Quality in Children with Therapy Resistant Epilepsy. Epilepsia. 2007;48(1):59-65. 